\title{
Vortex-antivortex dynamics and field-polarity-dependent flux creep in hybrid superconductor/ferromagnet nanostructures
}

\author{
M. Lange,* M. J. Van Bael, A. V. Silhanek, and V. V. Moshchalkov ${ }^{\dagger}$ \\ Nanoscale Superconductivity and Magnetism Group, Laboratory for Solid State Physics and Magnetism, K.U.Leuven, \\ Celestijnenlaan 200D, 3001 Leuven, Belgium \\ (Received 15 April 2005; published 22 August 2005)
}

\begin{abstract}
Vortex-antivortex arrays (VAA) that are created in a type-II superconducting film by lattices of ferromagnetic dots with perpendicular anisotropy have been investigated. The highest critical current is shifted to a nonzero value of the applied field, and current-voltage characteristics show different regimes of vortex motion even in zero-applied field due to the presence of the VAA. Creep of interstitial vortices is observed at low driving forces. This flux creep is strongly field-polarity dependent.
\end{abstract}

DOI: 10.1103/PhysRevB.72.052507

PACS number(s): 74.25.Qt, 74.25.Sv, 74.78.Fk, 74.78.Na

\section{INTRODUCTION}

In the mixed state, magnetic fields penetrate type-II superconductors in the form of vortices carrying a quantized magnetic flux, which normally corresponds to a single-flux quantum. ${ }^{1}$ In order to avoid dissipation in superconductors it is necessary to prevent vortex motion. Effective pinning can, for instance, be achieved by introducing columnar defects, ${ }^{2,3}$ lattices of submicron holes (antidots), ${ }^{4-7}$ or ferromagnetic dots. ${ }^{8-11}$ Besides enhanced critical currents, field-polaritydependent flux pinning was observed by Morgan and Ketterson in superconducting $\mathrm{Nb}$ films with arrays of ferromagnetic Ni dots, ${ }^{10}$ whose magnetization has an out-of-plane component. It was shown that in this system the critical current density $j_{c}$ depends on the mutual orientation of applied field and magnetization of the dots. Stable vortex-antivortex arrays (VAA) are predicted to exist in hybrid superconductor/ ferromagnetic systems even in zero external field due to the inhomogeneous stray fields of the ferromagnets. ${ }^{12-22}$ Despite strong interest in the theory of this subject, experimental reports on VAA have been very rare. ${ }^{23}$ Experiments on magnetic dots with out-of-plane magnetization have been carried out only for relatively weak stray fields in the sense that the stray field was screened by Meissner currents in the superconductor. ${ }^{24-26}$

In this paper we will show that the vortex dynamics in type-II superconducting $\mathrm{Pb}$ films is strongly influenced by the presence of ferromagnetic $\mathrm{Co} / \mathrm{Pd}$ dots with perpendicular anisotropy. We will characterize the dynamic behavior of stray field-induced VAA by means of current $(I)$-voltage $(V)$ characteristics and demonstrate pronounced field-polarity dependence of the flux creep. A clear sign for the existence of VAA is the presence of different regimes of vortex motion even in zero-applied field.

\section{EXPERIMENTAL}

Figure 1 shows a schematic drawing of the investigated sample. The structural and magnetic properties of this sample have been described elsewhere. ${ }^{27}$ The sample consists of a type-II superconducting $\mathrm{Pb}$ film (thickness $t_{P b}$ $=85 \mathrm{~nm}$, critical temperature $T_{c}=7.26 \mathrm{~K}$ ) evaporated on a
$1 \mathrm{~nm} \mathrm{Ge}$ base layer on a $\mathrm{Si}$ substrate with an amorphous $\mathrm{SiO}_{2}$ top layer. The $\mathrm{Pb}$ film is covered by a $10 \mathrm{~nm}$ Ge layer, which prevents the influence of the proximity effect with the metallic dot array. In order to avoid inhomogeneities of the current, this continuous $\mathrm{Ge} / \mathrm{Pb} / \mathrm{Ge}$ trilayer is patterned into a transport bridge (width $w=200 \mu \mathrm{m}$, distance between voltage contacts $d=630 \mu \mathrm{m})$ by optical lithography and chemical wet etching. Measurements of the upper critical field of this transport bridge allow an estimation of the coherence length $\xi(0)=34 \mathrm{~nm}$ and of the penetration depth $\lambda(0)$ $=49 \mathrm{~nm}$. The transport bridge is covered by ferromagnetic dots using electron-beam evaporation and electron-beam lithography. The dots consist of a 3.5-nm Pd base layer and a $[\mathrm{Co}(0.4 \mathrm{~nm}) / \mathrm{Pd}(1.4 \mathrm{~nm})]_{10}$ multilayer with perpendicular magnetic anisotropy. ${ }^{28}$ The dots are arranged in a square array with period $L=1.5 \mu \mathrm{m}$. They have a square shape with a side length of approximately $0.8 \mu \mathrm{m}$ with slightly irregular edges.

The vortex-pinning properties of this hybrid sample were investigated by electrical transport measurements for two different magnetic domain states of the $\mathrm{Co} / \mathrm{Pd}$ dots. After aligning the magnetic moments of the dots in positive $z$ direction $\left(m_{z}>0\right)$ (by magnetizing the dots in a high-positiveapplied field of $\mu_{0} H=+1 \mathrm{~T}$ perpendicular to the sample surface) the dots are in a single-domain state. ${ }^{27}$ After an ac demagnetization, the dots are in a multidomain state where the net magnetic moment of each dot $m$ is zero $(m=0) .{ }^{27}$ Note that the fields applied during all measurement are much weaker than the coercive field of the dot array at room temperature $\mu_{0} H_{c o e}=150 \mathrm{mT}$.

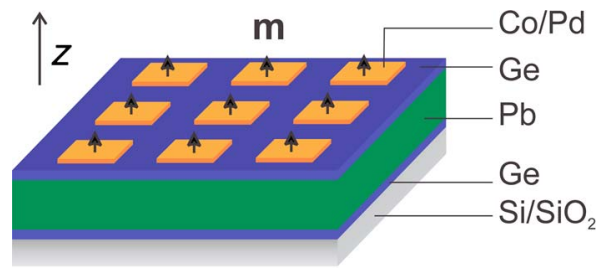

FIG. 1. (Color online) Schematic drawing of the investigated superconducting/ferromagnetic hybrid structure. The superconductor is a $85 \mathrm{~nm} \mathrm{~Pb}$ film, which is covered by an array of ferromagnetic $\mathrm{Co} / \mathrm{Pd}$ dots with out-of-plane magnetization. 

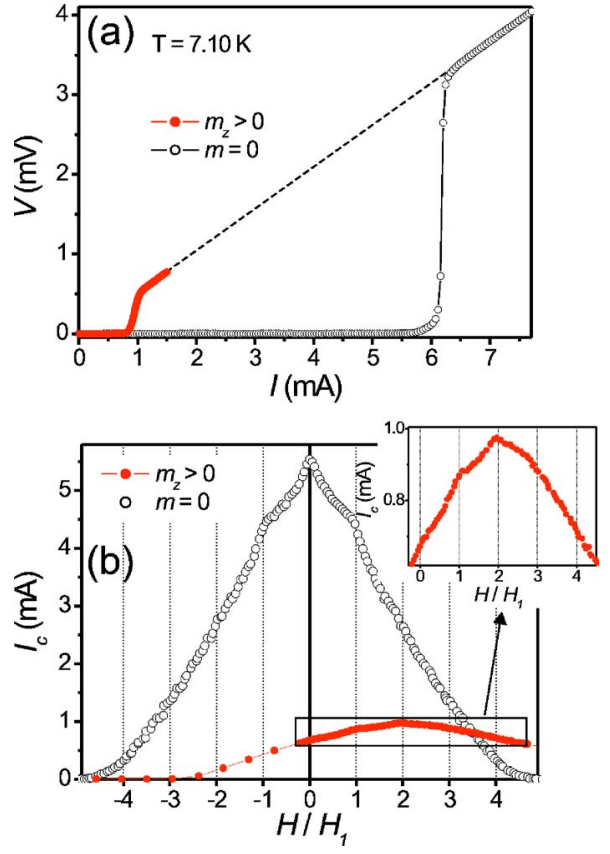

FIG. 2. (Color online) (a) $V(I)$ curves at $T=7.10 \mathrm{~K}$ in zero field after demagnetization $(m=0)$ and after magnetization of the sample in a high-positive field $\left(m_{z}>0\right)$. (b) Field dependence of the critical current of the superconductor with the dots in the two different magnetic states. A voltage criterion of $V_{c r}=1 \mu \mathrm{V}$ was used.

\section{CRITICAL CURRENT}

$V(I)$ curves of the sample are measured in a quantum design physical properties measurement system (PPMS) in standard four-point geometry by increasing $I$ starting from $I=0$. Figure 2(a) shows two $V(I)$ curves measured with the sample in the $m=0$ and the $m_{z}>0$ state at temperature $T$ $=7.10 \mathrm{~K}$ in zero field. There is a remarkable difference between the two curves: In the magnetized state, $V$ shows a steep transition to the normal state at about $6 \mathrm{~mA}$, while in the $m_{z}>0$ state this transition already appears around $1 \mathrm{~mA}$.

We define the critical current $I_{c}$ as the current where the voltage exceeds a certain voltage criterion $V_{c r}$. The critical current density $j_{c}$ can be calculated using $j_{c}=I_{c} /\left(t_{P b} w\right)$. The field dependence of $I_{c}$ at $T=7.10 \mathrm{~K}$ is shown for $V_{c r}=1 \mu \mathrm{V}$ in Fig. 2(b) for both magnetic states. The first matching field $H_{1}$ of this sample corresponds to the field value which generates one flux quantum per unit cell of the dot array and is given by $\mu_{0} H_{1}=\phi_{0} / L^{2}=0.92 \mathrm{mT}$ ( $\phi_{0}$ is the superconducting flux quantum). In the $m=0$ state, the maximum of $I_{c}$ is observed at zero field, and the $I_{c}(H)$ curve is symmetric with respect to $H=0$. Clear matching effects appear at positive and negative first-matching fields, indicating that the multidomain dots act as pinning sites for vortices due to the local suppression of the order parameter under the dots caused by the stray field, or due to the magnetic pinning of the vortices by the domain structure.

In the $m_{z}>0$ state, $I_{c}$ is lower than in the $m=0$ state for $H / H_{1}<3.5$. Surprisingly, the highest value of $I_{c}$ is not observed at $H=0$, but at $H / H_{1}=2$. Clear matching features are seen at $H / H_{1}=0,1$, and 2 . The strong overall reduction of

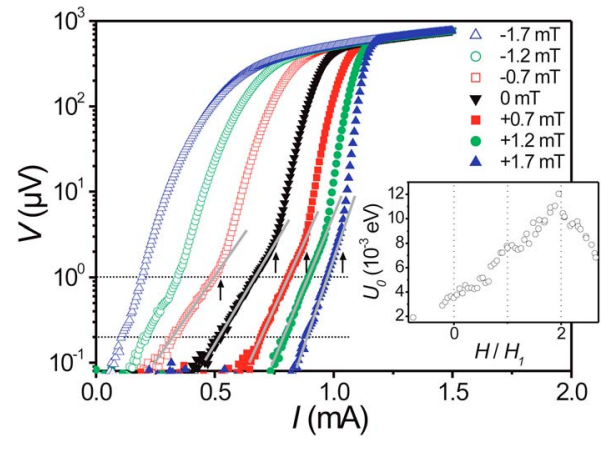

FIG. 3. (Color online) Field-polarity-dependent flux creep is observed in current-voltage characteristics of the superconductor at $T=7.10 \mathrm{~K}$. The dots are in the $m_{z}>0$ state and $V$ is plotted in logarithmic scale. The full lines are guides to the eye. The arrows indicate the crossover between low and high current regimes. The dotted lines indicate the used voltage criteria for determination of $I_{c}(H)$ (see text). The inset shows the field dependence of the activation energy $U_{0}$ determined from the slope of the $\ln V(I)$ curves.

the critical current indicates that the shift is not due to a mere compensation effect of the applied field by the local stray field of the dots, ${ }^{27}$ but that the vortex dynamics is strongly modified due to vortices induced by the dots, as will be explained below.

The significant alteration of $I_{c}(H)$, which is obtained only by a different magnetic history of the sample, must be related to the changed magnetic domain state of the dots. It should be noted that $H$ denotes only the externally applied field. The local stray field of the dots is also present at $H=0$. However, when integrated over the superconducting layer, the net average stray field of the dots will be near zero because of the returning stray field of opposite sign. For the further analysis of the data, we neglect the influence of heating effects, nonuniform current distributions, and surface barriers since they are the same in both magnetic states and cannot account for the observed significant change in $I_{c}(H)$.

In the following we will show that the existence of two vortices or one giant vortex with vorticity two directly beneath each dot can consistently explain the anomalous field shift and also other features in the $V(I)$ curves that will be discussed below. Additionally the returning stray field of the dots generates two antivortices per unit cell of the dot array in the superconductor. These antivortices are located at interstitial positions of the dot array. This assumption is justified by several theoretical calculations that predict this picture as a possible scenario. ${ }^{13-15,21}$ In the case of an isolated dot, both vortices and antivortices are bound to the dot, but for a regular array of dots the periodically modulated stray field could change this picture. If the spacing between the dots is sufficiently small, a motion of vortices and antivortices should be possible when a transport current exerts a sufficiently strong driving force on them.

\section{VORTEX DYNAMICS}

Figure 3 shows the $V(I)$ curves measured at $H=0$, $\pm 0.7 \mathrm{mT}, \pm 1.2 \mathrm{mT}$, and $\pm 1.7 \mathrm{mT}$ with $V$ plotted on logarith- 
mic scale. Low and high current regimes must be distinguished: In the low current regime only interstitial flux lines move, whereas at higher applied current both interstitial flux lines and the ones that were originally pinned on the dot sites move. Different dynamic regimes for low and high currents have also been observed in $V(I)$ curves of superconducting films with arrays of antidots ${ }^{29}$ and arrays of magnetic dots. ${ }^{30}$ Thus, the $V(I)$ curve at $H=0$ is in agreement with the assumption that interstitial antivortices are present even in zero field.

In the low current region $(V \lesssim 5 \mu \mathrm{V})$ the $\ln V(I)$ curves measured in zero field and positive fields have a linear slope as is indicated by the straight lines. This linear slope is less clear for the curve at $-0.7 \mathrm{mT}$ and disappears as $H$ is further decreased. A linear $\ln V(I)$ dependence is predicted in the Anderson and Kim model for flux creep. ${ }^{31}$ In this model the electric field $E$ generated by the vortices creeping from one pinning site to another in the limit $I \approx I_{c}$ is given by

$$
E \propto e^{\left(U_{0} / k_{B} T\right)\left[\left(I / I_{C}\right)-1\right]}, \quad I \approx I_{c},
$$

with $k_{B}$ the Boltzmann constant and $U_{0}=U_{0}(T, H)$ the activation energy, i.e., the height of the potential barrier between two adjacent pinning sites. Thus,

$$
\ln V=\frac{U_{0}}{k_{B} T}\left(\frac{I}{I_{c}}-1\right)+C(H, T)
$$

where $\mathrm{C}$ is independent of $I$. This means that in zero-applied field the experimentally observed $\ln (V) \propto I$ behavior is consistent with a creep of antivortices over the potential barriers that are formed by the dots and the induced vortices. Hence, Fig. 3 shows that flux creep in hybrid superconductor/ ferromagnet systems is strongly dependent on the field polarity. The exact creep pattern of the antivortices in the sample remains an open question. An antivortex can, for example, move by annihilating first with a vortex on the edge of a dot, and at the same time a new antivortex is generated at the opposite dot edge. Another possibility is that the antivortices creep through channels between the dots. A theoretical treatment in the framework of the time-dependent GinzburgLandau theory is required to answer this question.

The slope of the $\ln V(I)$ curves is only determined by $U_{0}, T$, and $I_{c}$. For the calculation of $U_{0}$ (results are shown in the inset of Fig. 3), we have defined $I_{c}$ with a criterion of $V_{c r}=0.2 \mu \mathrm{V}$. Up to $H_{2}$, both $U_{0}$ and $I_{c}$ increase with $H$, which is related to the decreasing number of antivortices. As the number of antivortices goes down, the effective barrier that they have to overcome is increased due to a decreasing mutual repulsive interaction. Note that this interaction is not included in the model (in its simplest interpretation). At $H_{1}$ a peak in $U_{0}(H)$ is observed because of the commensurability of the antivortices with the periodic pinning potential. Above $\mathrm{H}_{2}, \mathrm{U}_{0}$ starts to decrease drastically as $\mathrm{H}$ is increased.

This crossover can be explained by the appearance of vortices at interstitial positions of the dot array: the number of vortices increases and therefore $U_{0}$ decreases with increasing $H$. The most important conclusion of these results is that the observed features are in good agreement with the assumption that at $H=0$, two antivortices per unit cell are induced in the

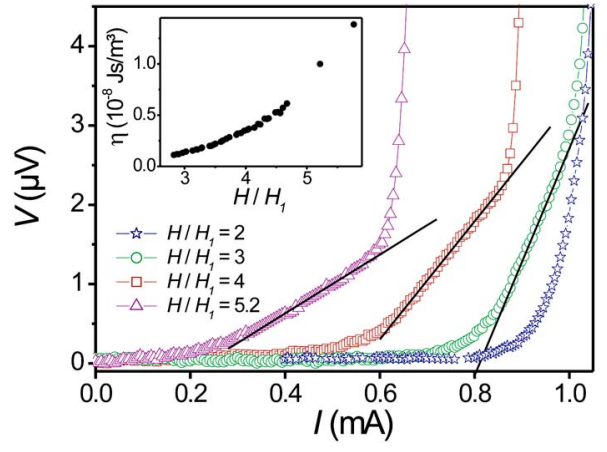

FIG. 4. (Color online) Current-voltage characteristics of the superconductor at $T=7.10 \mathrm{~K}$ in the $m_{z}>0$ state at $H / H_{1}=2,3,4$ and 5.2 for small voltages. The slope of the straight lines determines the viscous drag coefficient $\eta$ in the flux-flow regime $H>2.8$.

superconductor at interstitial positions of the dot array. Small inhomogeneities of the dots in a real sample could lead to a variation of the stray fields of the dots, which for a small fraction of dots could result in three or only one induced vortex-antivortex pairs instead of two. However, this will not influence the general behavior of the sample.

A crossover to a linear $V(I)$ dependence is observed for $H / H_{1}>2.8$. The main panel in Fig. 4 shows the low-voltage part of the $V(I)$ curves in the $m_{z}>0$ state for $H / H_{1}=2,3,4$, and 5.2. The linear dependence is consistent with the situation that local pinning forces are overcome, resulting in a steady motion of the interstitial vortices ${ }^{32}$ with velocity $v$ given by

$$
\eta v=\left(I-I_{c}\right) \phi_{0} / t_{P b} w,
$$

with $\eta$ a viscous drag coefficient, which can be determined from

$$
V=E d=n_{f} \phi_{0} v d=n_{f} \phi_{0}^{2} d\left(I-I_{c, \text { flow }}\right) / \eta t_{P b} w,
$$

where $n_{f}$ is the areal density of moving interstitial vortices given by $n_{f}=\mu_{0}\left|H-H_{2}\right| / \phi_{0} . I_{c, \text { flow }}$ is defined as the value of $I$ where the straight lines characterizing the flux-flow regime indicated in Fig. 4 cross the $I$ axis. From the slope of the $V(I)$ curves and the value of $I_{c, \text { flow }}, \eta(H)$ is calculated using Eq. (4); see the inset of Fig. 4. In the Bardeen-Stephen model for free-flux flow, ${ }^{33}$ it is assumed that $\eta$ does not depend on the applied field. The flux-flow phase of the interstitial vortices in our measurements can therefore not be interpreted in terms of free-flux flow. The mutual interaction between flowing interstitial vortices and pinned vortices on the dot sites cannot be neglected and should be the main reason for the increase of $\eta$ as $H$ is increased.

Finally, we would like to point out that ferromagnetic dots can be very useful for the new field of fluxonics. Here the aim is to guide the vortices through nanoengineered arrays of pinning sites or channels in the superconductor, ${ }^{32}$ which makes it possible to develop new devices, like superconducting reversible rectifiers ${ }^{30}$ or ratchet cellular automata based on nanostructured superconductors. ${ }^{34}$ Using VAA in a welldesigned fashion makes it possible to operate fluxonic devices without exposing them to externally applied fields. For 
instance, by using triangular-shaped magnetic dots it is possible to measure dc voltages upon application of an ac current $^{30}$ even in zero field. Furthermore, by using rectangular dots that generate one vortex-antivortex pair per dot, logic elements can be developed ${ }^{34}$ where the 0 and 1 states are defined by the position of the antivortex.

\section{CONCLUSION}

In conclusion, vortices in hybrid superconductor/ ferromagnet nanostructures can have peculiar dynamic properties due to the presence of the vortex-antivortex arrays that are induced in the superconductor by the inhomogeneous stray field of the ferromagnet. In zero-applied field, two re- gimes of vortex motion are observed, which proves the existence of these induced vortex-antivortex arrays. In the lowcurrent regime, field-polarity-dependent flux creep has been demonstrated.

\section{ACKNOWLEDGMENTS}

The authors are thankful to E. Claessens for help with the measurements, to S. Raedts, M. Morelle, and K. Temst for their contribution to the sample preparation, and to $\mathrm{Y}$. Bruynseraede for fruitful discussions. This work was supported by the Belgian IUAP, the ESF "VORTEX," and the K. U. Leuven Research Fund GOA/2004/2 programs, and by the Fund for Scientific Research-Flanders (Belgium) (F.W.O.-Vlaanderen).
*Present address: nv Bekaert sa, Bekaertstraat 2, 8550 Zwevegem, Belgium

†Electronic address: victor.moshchalkov@fys.kuleuven.ac.be

${ }^{1}$ A. A. Abrikosov, Sov. Phys. JETP 5(6), 1174 (1957).

${ }^{2}$ L. Civale, A. D. Marwick, T. K. Worthington, M. A. Kirk, J. R. Thompson, L. Krusin-Elbaum, Y. Sun, J. R. Clem, and F. Holtzberg, Phys. Rev. Lett. 67, 648 (1991).

${ }^{3}$ J. R. Thompson, L. Krusin-Elbaum, L. Civale, G. Blatter, and C. Feild, Phys. Rev. Lett. 78, 3181 (1997).

${ }^{4}$ A. F. Hebard, A. T. Fiory, and S. Somekh, IEEE Trans. Magn. 1, 589 (1977).

${ }^{5}$ M. Baert, V. V. Metlushko, R. Jonckheere, V. V. Moshchalkov, and Y. Bruynseraede Phys. Rev. Lett. 74, 3269 (1995).

${ }^{6}$ V. Metlushko, U. Welp, G. W. Crabtree, Zhao Zhang, S. R. J. Brueck, B. Watkins, L. E. DeLong, B. Ilic, K. Chung, and P. J. Hesketh, Phys. Rev. B 59, 603 (1999).

${ }^{7}$ C. C. D. Silva, L. R. E. Cabral, and J. A. Aguiar, Phys. Status Solidi A 187, 209 (2001).

${ }^{8}$ O. Geoffroy, D. Givord, Y. Otani, B. Pannetier, and F. Ossart, J. Magn. Magn. Mater. 121, 223 (1993).

${ }^{9}$ J. I. Martín, M. Veléz, J. Nogués, and I. K. Schuller, Phys. Rev. Lett. 79, 1929 (1997).

${ }^{10}$ D. J. Morgan and J. B. Ketterson, Phys. Rev. Lett. 80, 3614 (1998).

${ }^{11}$ M. J. Van Bael, K. Temst, V. V. Moshchalkov, and Y. Bruynseraede et al., Phys. Rev. B 59, 14674 (1999).

${ }^{12}$ I. K. Marmorkos, A. Matulis, and F. M. Peeters, Phys. Rev. B 53, 2677 (1996).

${ }^{13}$ M. V. Milošević, S. V. Yampolskii, and F. M. Peeters, Phys. Rev. B 66, 024515 (2002).

${ }^{14}$ M. V. Milošević and F. M. Peeters, Phys. Rev. B 68, 024509 (2003)

${ }^{15}$ M. V. Milošević and F. M. Peeters, Phys. Rev. Lett. 93, 267006 (2004).

${ }^{16}$ Y. I. Bespyatykh and W. Wasilevski, Phys. Solid State 43, 224 (2001).
${ }^{17}$ S. Erdin, I. F. Lyuksyutov, V. L. Pokrovsky, and V. M. Vinokur, Phys. Rev. Lett. 88, 017001 (2002).

${ }^{18}$ S. Erdin, A. F. Kayali, I. F. Lyuksyutov, and V. L. Pokrovsky, Phys. Rev. B 66, 014414 (2002).

${ }^{19}$ A. Y. Aladyshkin, A. S. Mel'nikov, and D. A. Ryzhov, J. Phys.: Condens. Matter 15, 6591 (2003).

${ }^{20}$ R. Laiho, E. Lähderanta, E. B. Sonin, and K. B. Traito, Phys. Rev. B 67, 144522 (2003).

${ }^{21}$ D. J. Priour, Jr. and H. A. Fertig, Phys. Rev. Lett. 93, 057003 (2004).

${ }^{22}$ G. Carneiro, Phys. Rev. B 69, 214504 (2004).

${ }^{23}$ M. J. Van Bael, J. Bekaert, K. Temst, L. Van Look, V. V. Moshchalkov, Y. Bruynseraede, G. D. Howells, A. N. Grigorenko, S. J. Bending, and G. Borghs, Phys. Rev. Lett. 86, 155 (2001).

${ }^{24}$ M. J. Van Bael, L. Van Look, K. Temst, M. Lange, J. Bekaert, U. May, G. Güntherodt, V. V. Moshchalkov, and Y. Bruynseraede, Physica C 332, 12 (2000).

${ }^{25}$ M. J. Van Bael, M. Lange, S. Raedts, V. V. Moshchalkov, A. N. Grigorenko, and S. J. Bending, Phys. Rev. B 68, 014509 (2003).

${ }^{26}$ A. N. Grigorenko, S. J. Bending, M. J. Van Bael, M. Lange, V. V. Moshchalkov, H. Fangohr, and P. A. J. de Groot, Phys. Rev. Lett. 90, 237001 (2003).

${ }^{27}$ M. Lange, M. J. Van Bael, Y. Bruynseraede, and V. V. Moshchalkov, Phys. Rev. Lett. 90, 197006 (2003).

${ }^{28}$ P. F. Carcia, A. D. Meinhaldt, and A. Suna, Appl. Phys. Lett. 47, 178 (1985).

${ }^{29}$ E. Rosseel, M. J. Van Bael, M. Baert, R. Jonckheere, V. V. Moshchalkov, and Y. Bruynseraede, Phys. Rev. B 53, R2983 (1996).

${ }^{30}$ J. E. Villegas, S. Savel'ev, F. Nori, E. M. Gonzalez, J. V. Anguita, R. Garcia, and J. L. Vicent, Science 302, 1188 (2003).

${ }^{31}$ P. W. Anderson and Y. B. Kim, Rev. Mod. Phys. 36, 39 (1964).

${ }^{32}$ A. V. Silhanek, L. Van Look, S. Raedts, R. Jonckheere, and V. V. Moshchalkov, Phys. Rev. B 68, 214504 (2003).

${ }^{33}$ J. Bardeen and M. J. Stephen, Phys. Rev. 140, A1197 (1965).

${ }^{34}$ M. B. Hastings, C. J. Olson Reichhardt, and C. Reichhardt, Phys. Rev. Lett. 90, 247004 (2003). 